Int. J. Dev. Biol. 48: 755-760 (2004)

doi: $10.1387 / \mathrm{ijdb} .041881 \mathrm{rn}$

\title{
The little $R$ cell that could
}

\author{
RAGHAVENDRA NAGARAJ and UTPAL BANERJEE*
}

Department of Molecular, Cell and Developmental Biology, Department of Biological Chemistry and Molecular Biology Institute. University of California, Los Angeles, CA USA

\begin{abstract}
Drosophila eye development provides an excellent model system to study the role of inter-cellular signaling in the specification of unique cell fates. Behavioral screens by Benzer and his colleagues led to the identification of a gene, Sevenless, a receptor tyrosine kinase (RTK) receptor, required for the specification of the UV sensitive R7 cell. Genetic analysis further showed that the Ras/Raf/MAPK pathway function downstream of Sevenless in the specification of R7 fate. Signaling mediated by another RTK, EGFR and Notch have also been shown to function in either an antagonistic or a synergistic manner in the specification of cell fate during eye development. In some instances, these pathways are linked in a sequential manner by the regulation of the expression of Notch ligand, Delta by EGFR, while in others, these pathways function in a combinatorial fashion on enhancer elements to control target gene expression. In this review, we highlight the elegant genetic strategies used by several laboratories in early elucidation of the Sevenless pathway which helped link the RTK receptor to the Ras/Raf/MAPK cascade and discuss how EGFR and Notch signaling pathways are used in a reiterative manner and by combining in different modes, generate sufficient diversity required for the specification of unique cell fates.
\end{abstract}

KEY WORDS: RTK, Sevenless, Son of Sevenless, Ras, Notch

\section{Introduction}

Intercellular signaling plays a critical role in the establishment of the body plan and cell fate during the development of multicellular organisms. Even though the variation in cell fates adopted is quite extensive, only a limited number of conserved signaling pathways mediate the majority of cell-cell interactions. This paradox is addressed in part by the observation that individual signaling pathways function in multiple settings and combinations to establish unique cell fates. The unique response to a signal in part depends on its amplitude, its duration and in the pre-patterning of the cell receiving the signal. Understanding how this context is created within an equipotent group of cells is fundamental to our understanding of the role of cell signaling in fate specification during development.

The Drosophila compound eye consists of a large number of ommatidia (facets), each containing a fixed number of cells: eight photoreceptor cells ( $R$ cells), four non-neuronal cone cells, three classes of pigment cells and a bristle complex. The Drosophila eye develops from a sheet of epithelial tissue called the eye imaginal disc. In the third larval instar, an indentation called the morphogenetic furrow (MF) initiates at the posterior tip and sweeps anteriorly across the disc. As cells emerge out of the furrow, they attain competence to initiate differentiation in a precise order (Fig. 1).

The photoreceptors are the first cells to differentiate followed by the non-neuronal cone and pigment cells. The Drosophila eye and antenna both arise from a single primordium at stage 14-15 of embryonic development, and only later the posterior region of this common primordium differentiates into the eye disc while the anterior region gives rise to the antenna (Wolff and Ready 1993). Inter-cellular signaling mediated by the Notch and the EGFR pathways have been proposed to function in the establishment of eye vs antennal fate (Kumar and Moses 2001. Kenyon et.al 2003). This interplay of signals is important for the expression of the Eyeless (DPax-6) protein in the aspect of the tissue that will give rise to the eye (Onuma et. al. 2002). Further specification is then achieved by a network of transcriptional regulators involving multiple feedback regulatory loops that reinforce each other's expression and control the program of eye fate establishment (Pappu and Mardon 2002). Since all the developmental decisions in the eye are mediated by inter-ceullar signaling, the Drosophila

Abbreviations used in this paper: Boss, bride of sevenless gene; EGFR, epidermal growth factor receptor; MAPK, map kinase; MF, morphogenetic furrow; RTK, receptor tyrosine kinase.

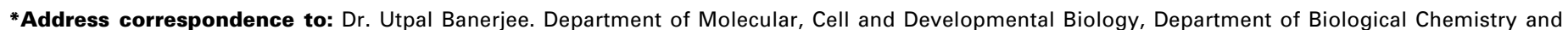
Molecular Biology Institute. University of California, Los Angeles, CA 90095. Fax: +1-310-206-9062. e- mail: banerjee@mbi.ucla.edu
} 
eye has been long considered an important model system for studying how signaling pathways influence cell fate. In this review, we briefly discuss the history of events that led to some of these ideas. This is by no means a comprehensive description of eye development for which there are many excellent recent reviews (K. Moses (Ed) 2002). Here we discuss the genetic dissection tools that helped identify critical functional pathways and established the logic by which signaling pathways function in the specification of unique cell fate.

\section{Lack of clonality in cell fate specification}

Waddington and Perry's light and electron microscopic studies laid the foundation for our understanding of the organization of different cell types within a single ommatidium of the Drosophila compound eye (Perry, 1968; Waddington and Perry, 1961). In their pioneering work, they established the number and precise morphological features of each of the cell types in the eye and described their overall organization within each facet of the adult compound eye. Developmental models that explain how this pattern is generated had an earlier but shaky start. Bernard (1937) had proposed the ommatidial stem cell model based on sections of the compound eye of the ant, Formicina. According to this model, all cells within the ommatidium are related clonally with a stem cell undergoing three rounds of cell division to generate the 8 photoreceptor cell types. Subsequently, Baker (1963) and Shoup (1966) supported this view after studying gross pigmentation patterns in the eye. The insect eye, in this view, was primitive and non -plastic relying on a simple sequence of clonal events to achieve its final form.

Benzer and coworkers (Hotta and Benzer, 1969; Hotta and Benzer, 1970; Ready et al., 1976) using gynandromorphs and somatic mosaic clones, and later Lawrence and Green (1979), using small two cell clones, definitively established the absence of any clonal relationship between the cells of the facet. In the earliest study, Hotta and Benzer (1970) showed that in clones of the retinal degeneration mutation $r d g B$, a mixture of mutant and non mutant cells can constitute an individual facet. This suggested that the photoreceptor cell types within a given facet could not have arisen clonally. Don Ready (Ready et al., 1976) further extended these observations using somatic recombination to mark clones with a pigmentation marker, white, which is essential for the coloration of pigment granules. At the boundaries between pigmented and non- pigmented regions each ommatidium contained a mixture of cell types that either contained or lacked pigmentation. These observations proved that the cells within a single ommatidium are derived in a non-clonal manner. These results also established the Drosophila eye as a premier model system for understanding cell-cell interaction and the important role this process plays in the specification of cell fate.

Waddington and Perry's cytological studies suggested that the photoreceptors are divided into two broad groups based on their position within the facet and the size of the rhabdomere (the light sensing region of the photoreceptor). The outer photoreceptors (R1-R6) have large rhabdomeres and form an outer ring, while the $\mathrm{R} 7$ and $\mathrm{R} 8$ cells have small rhabdomeres and are localized in the central region of the facet. Apart from the size and the location of their rhabdomere, the outer and the inner photoreceptors also differ in the spectral sensitivities of their Rhodopsins (Harris and Stark, 1977; Harris et al., 1976). In particular, the R7 cell senses UV radiation, a property that was later very useful in identifying mutants that lack this cell type. Using phototaxis assays, Harris and Stark (Harris et al., 1976) identified mutations, which specifically affect certain types of photoreceptor function or development. In the retinal degeneration B (rdgB) (Alawi et al., 1972; Hotta and Benzer, 1970) mutant the outer photoreceptors degenerate upon exposure to light whereas the inner ones remain functional. Later studies demonstrated that genes of the rdg family encode for proteins that specifically function in the outer photoreceptor cells in phototransduction (Pak and Leung, 2003). Using EMS mutagenesis, Koeing and Merriam (1975) identified ora mutations in which the outer photoreceptor cells fail to develop. These observations suggested that an independent genetic program regulates the establishment and function of the outer and inner photoreceptor cells in the Drosophila eye.

\section{Sevenless pathway}

Phototaxis assays developed by Harris, Stark and Benzer led to the identification of a large number of developmentally important genes required for the fate and function of photoreceptor cells. One such novel mutation was sevenless (sev) in which the R7 photoreceptor is missing from each ommatidium. Tomlinson and Ready (1986) were the first to report a detailed characterization
Fig. 1. A schematic representation of cell fate specification events during Drosophila eye development. Cells anterior to the Morphogenetic Furrow (MF) are undifferentiated. As the cells emerge out of the furrow they become competent to undergo differentiation. The photoreceptor cells are the first to differentiate followed by the nonneuronal cone cells and pigment cells.

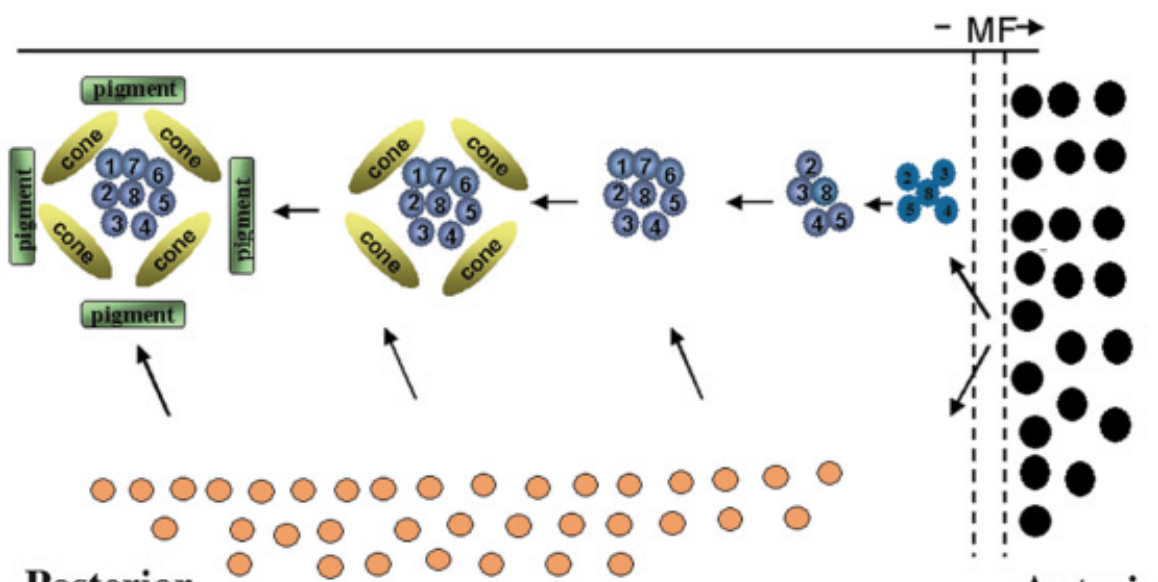

Posterior 
of the sevmutation. Using histological sections of third instar eye discs they showed that in sev mutants the precursor of the R7 neuron assumes a non-neuronal cone cell fate. They also established that a synchronized apical movement of nuclei preceded each cell fate decision within an ommatidium (Tomlinson and Ready, 1987). In sevmutants, as in wild type, the nucleus of the pre-R7 cell initiates nuclear migration and moves to the apical surface but fails to move basally at the proper time and instead assumes the fate of a cone cell (Tomlinson and Ready, 1986). Mosaic analysis further suggested that the' sevgene functions in a cell-autonomous manner, i.e. it is required within the $\mathrm{R} 7 \mathrm{cell}$ to specify its own fate (Tomlinson and Ready, 1987). The sevgene was cloned independently by the Rubin (Hafen et al., 1987) and Benzer (Banerjee etal., 1987a; Banerjee etal., 1987b) laboratories. Sev is a receptor tyrosine kinase and its activation induces intracellular changes in the presumptive R7 cell that causes it to adopt an R7 rather than a cone cell fate (Basler and Hafen, 1988).

The expression of Sevenless is not restricted to the presumptive R7 cell (Tomlinson et. al. 1987, Banerjee et al., 1987b). Electron microscopy as well and the analysis of the sevenhancer suggested that Sevenless is expressed in R3/R4, R7, R1/R6 and cone cells (Tomlinson et. al. 1987). Thus the expression pattern of Sevenless did not provide the explanation for its specific effects on a single cell within the ommatidium. Rosemary Reinke in Larry Zipursky's laboratory described a mutant in which $\mathrm{R} 7$ cells were missing but unlike sev, the function of the gene was found to be required in the neighboring R8 cell. Reinke and Zipursky (1988) named this mutant bride of sevenless (boss). Later studies showed that boss encodes a seven-pass transmembrane protein that is specifically expressed in the apices of the R8 cell and functions as a ligand for the Sevenless receptor (Hart et al., 1990). The tight spatial and temporal regulation of boss is important for the pre-R7 cell to receive the Sevenless signal and adopt the $\mathrm{R} 7$ fate (Van Vactor et al., 1991).

Homozygous mutations in both sev and boss are viable, showing only a loss of R7 cells in the eye. However, genes functioning downstream of the Sevenless pathway were expected to function in other tyrosine kinase pathways and their loss of function could likely lead to early lethality. To overcome this problem several laboratories used sensitized genetic screens to identify downstream components of the Sevenless pathway. These screens were based on the premise that when the signal transmitted by a pathway is genetically decreased to a level close to the threshold of wild-type function, the phenotype becomes susceptible to the dosage of other genes that participate in the pathway. Thus, inactivation of a single copy of a gene related to the pathway or modest overactivation of a downstream component in a sensitized genetic background would cause phenotypically detectable changes in the level of the signal. As only one of the two copies of this second gene is eliminated, there is no lethality associated with such genetic combinations. Using this method it became possible to identify downstream components of Sevenless and other RTK pathways.

While still a post doctoral fellow with Seymour Benzer, one of us (U.B.) conducted a second-site dominant suppressor screen to identify downstream components of the Sevenless pathway using an allele of $\operatorname{sev}, \operatorname{sev}^{\mathrm{E} 4}$, which encodes a full sized protein but is functionally defective and does not support $R 7$ specification. A gain of function mutation which partially suppressed the sev E4

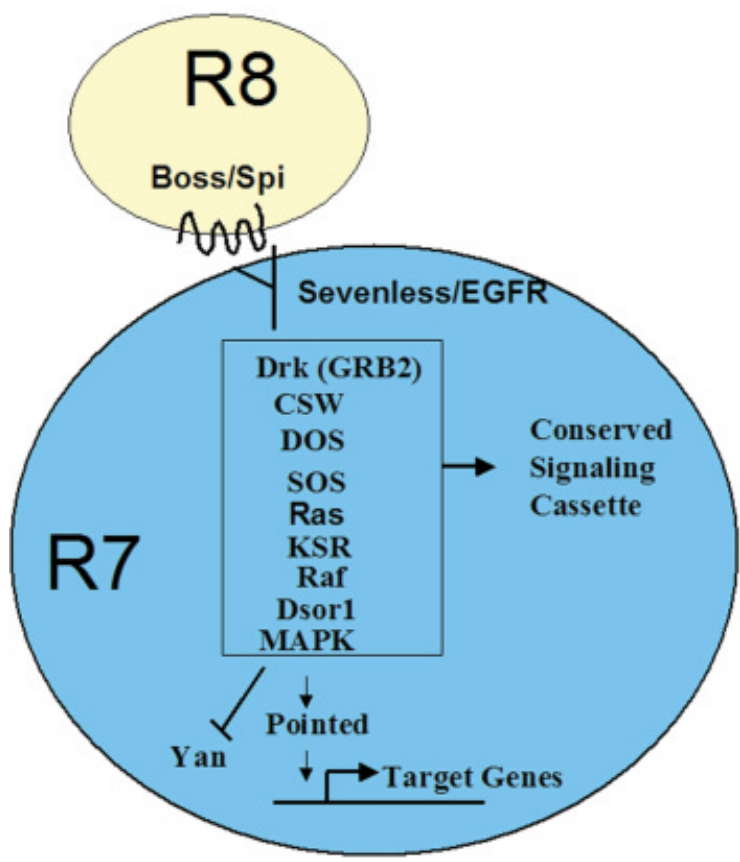

Fig. 2. The $R 8$ cell signals to the precursor of the $R 7$ cell via the Sevenless pathway to promote $\mathbf{R} 7$ fate. Bride of Sevenless (Boss), the ligand for the Sevenless receptor, is expressed in the R8 cell. Binding of Boss to Sev causes upregulation of RTK activity of Sev, resulting in the activation of Ras by the Grb2/Sos complex. Activated Ras, in-turn, promotes the activation of the kinase cascade culminating in the activation and translocation of MAPK into the nucleus. Phosphorylation of key substrates by MAPK in the nucleus promotes the transcriptional upregulation of target genes necessary for specification of $R 7$ fate. The EGFR pathway shares many of the components which function in the Sevenless pathway.

phenotype and partially restores $\mathrm{R} 7$ specification was identified in this screen and was named son of sevenless (sos) (Rogge et al., 1991). This gene was also identified by Simon et. al. (1991) in their screen for enhancers of a temperature sensitive sevenless allele (see below). The identification of the same gene in independent screens as a suppressor and enhancer of sevsuggested that sos plays a central role in the Sevenless signal transduction pathway. Simon et. al. (1991) and Bonfini et. al. (1992) cloned sos and showed that it encodes a conserved protein similar to CDC25 in $S$. cerevisiae, which functions as a guanine nucleotide exchange factor (GEF). This was the first example of a GEF in higher eukaryotes that promotes the activation of Ras by converting the GDP bound inactive to the GTP bound active form. Studies in vertebrate systems had long identified RTK signaling and Ras proteins as major players in the development of cancer. However the identification of sos in Drosophila was the first study to demonstrate the linear activation of Ras by an RTK via the mediation of Sos.

Simon et. al. (1991) and Basler et. al. (1991) developed elegant strategies to look for other components of the Sevenless pathway. Simon et. al. (1991) generated a temperature sensitive allele of sev, sev ${ }^{\mathrm{B} 4}$ by introducing an amino acid change in a residue conserved between $v$-src and sev. They showed that $\operatorname{sev}^{\mathrm{B} 4}$ flies 


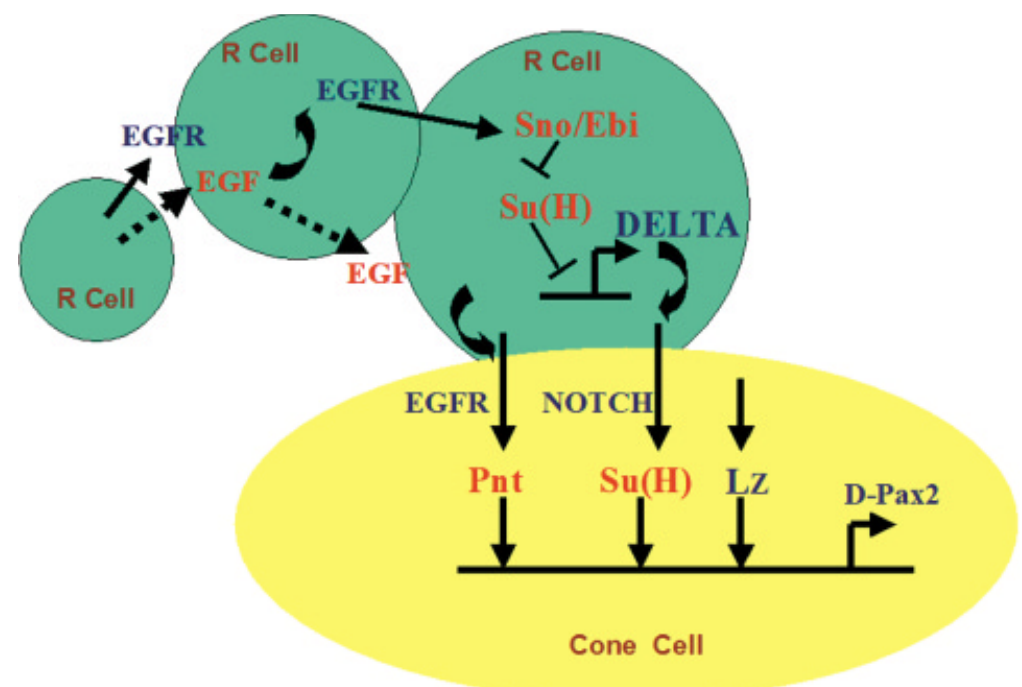

Fig. 3. Sequential and combinatorial integration of the EGFR and Notch pathways in the specification of cone cell fate. In the $R$ cells, activation of the Notch ligand, Delta requires high levels of EGFR signaling. Delta in the $R$ cells signals the neighboring undifferentiated cells and causes the activation of the Notch pathway. These cells also receive an EGFR signal from the $R$ cells and express Lozenge. A combinatorial integration of EGFR, Notch and Lz pathways in the undifferentiated cells results in the activation of D-Pax2 and their differentiation into cone cell.

are largely wild type at the permissive temperature and largely lacking R7's at non-permissive temperatures. These observations suggested that the mutant $\mathrm{Sev}^{\mathrm{B} 4}$ protein functions at a threshold level and a single copy loss of any of the downstream components of the pathway would weaken the Sev signal to below this threshold. Using this system they conducted a dominant second site suppressor screen and identified 5 distinct loci that encode downstream components. These included Ras and Sos as well as Drk, an adaptor protein homologous to Grb2 (Koch et al., 1991) with $\mathrm{SH} 2$ and $\mathrm{SH} 3$ domains. Drk and was later shown to physically link RTKs to Sos (Simon et al., 1991; Simon et al., 1993). GAP1 was identified in sensitized screens as a negative regulator of the RTK pathway (Gaul et al., 1992; see also Rogge et al., 1992).

Hafen and coworkers (Basler etal., 1991; Dickson et al., 1992) used a slightly different approach to generate a sensitized genetic system to look for genes downstream of the Sevenless pathway. They induced a mutation within the catalytic domain of Sev which made it constitutively active. Under the control of the sevenhancer, transgenic flies expressing this receptor show conversion of nonneuronal cone cells into photoreceptor neurons (Basler et al., 1991). They conducted a dominant suppressor screen to identify genes which suppress this phenotype and identified mutations in genes encoding Drk and Raf, a Serine/Threonine kinase, as downstream suppressors (Dickson et al., 1992; Olivier et al., 1993). Further, the Hafen and Zipursky laboratories extended this approach by creating a constitutively active version of Raf and identified rolledas a mutant allele of a gene encoding Drosophila MAPK (Biggs et al., 1994; Biggs and Zipursky, 1992; Brunner et al., 1994). Screens for dosage sensitive modifiers using hyperactive components of the RTK pathway further resulted in the identification of many members of the pathway including cytoplasmic and nuclear components (Fig. 2).

\section{Signal integration and cell fate}

The Sevenless pathway was so exclusively involved in R7 development that it raised the question whether different cell fates within the Drosophila compound eye might each require a unique cell signaling pathway for its fate specification. From the outset it was obvious that this could not be the case since all cell fate specification events in the eye require signaling mediated by the EGFR pathway (Baker and Rubin, 1989; Baker and Rubin, 1992; Freeman, 1996). Later observations established that the differential response to a given signal appears to lie in its ability to combine with other signals in generating a unique response. The assumption of specific fates in the second wave of morphogenesis in the eye that results in the differentiation of $R 1, R 6, R 7$, cone cells and pigment cells (Wolff and Ready, 1991), has been studied in much detail to arrive at this combinatorial model for signaling (Flores et al., 2000; Xu et al., 2000). A single pool of undifferentiated cells gives rise to all these different cell fates manifested by the expression of cell type specific transcription regulators (Kumar and Moses, 1997). The expression of these cell type specific factors has been shown to require the function of the EGFR and Notch signals as well as the Runt-domain containing transcription factor Lozenge. How do these multifunctional signaling pathways function in the specification of unique cell fates and activate cell type specific factors? The EGFR and the Notch pathways have been shown to combine in both a sequential (Tsuda et al., 2002) and in a parallel fashion (Flores et al., 2000) in unique combinations to regulate transcriptional regulators. For example, the activation of the $D$-Pax2enhancer in cone cells requires the input of the EGFR and Notch pathways and the transcription factor, Lozenge. Even though Lz is expressed all in the precursor cells from which cone cells are derived, the activation of D-Pax2 is limited to the cells that differentiate as cone cells. This is because a optimal combination of activated transcription factors downstream of EGFR and Notch must directly combine with Lz on the D-Pax2 enhancer for limiting D-Pax2 expression to the cone cells (Fig. 3).

Each cell type in the eye does not have its own unique signal but makes use of a combinatorial code involving a very small number of common signals. For example, a proper combination of Notch and EGFR in the presence of $L z$ is able to define the fates of all cells arising after the five cell precluster (Flores et al., 2000). Since the downstream components of Notch and EGFR are present in all cell types, the precise activation of a given pathway depends upon the spatio-temporally restricted presentation of the ligand (Freeman, 1996; Tsuda et al., 2002). The activation of Notch in the cone cells requires the expression of the ligand, Delta in photoreceptor cells. Delta expression in the R cells in turn requires the function of EGFR (Tsuda et.al 2002). Interestingly, the regulation of Delta by EGFR involves the proteasome complex and is regulated by controlling the nuclear localization of the corepressor protein, SMRTER. Thus, the specification of R-cell fate and the activation of Delta in R-cells both need EGFR, but different downstream branches of the pathway are used, presumably to set different thresholds for these independent functions. In R-cells, the EGFR and the Notch pathway are first 
linked in a serial manner and then subsequently these pathways combine in parallel to signal their neighbors to promote cone cell differentiation (Fig. 3). This interlinking of signaling pathways in different modes introduces temporal delays in the system allowing each cell type to follow its unique path of differentiation. What is remarkable about this system, likely to be repeated in mammalian development, is that the timing and combination of a very small number of signals can produce a great diversity of developmental outcomes.

\section{Conclusion}

The pioneering work of Benzer and his early coworkers laid the foundation for the genetic analysis of eye development in Drosophila. Their work first suggested that short range intercellular signaling is at the core of all cell fate specification events during eye development. Later, Rubin, Benzer and their intellectual descendents were largely responsible for the unraveling of the RTK pathway in the context of the development of a single cell type, the R7 photoreceptor. During the time when the Sevenless pathway was being unraveled in flies, studies in the worm $C$. elegansled to the independent identification of the RTK signaling system in the context of cell fate specification (Aroian et al., 1990; Han et al., 1993; Han and Sternberg, 1990; Hill and Sternberg, 1992). These studies, complemented by biochemical analysis in mammalian systems, led to the establishment of the now well established paradigm in which activation of RTK signal results in the activation of the Ras/Raf/MAPK cascade and eventual phosphorylation of key targets within the nucleus (Pawson, 2002). The principles guiding the establishment of the $R 7$ fate also laid the foundation for understanding how developmental signals combine in the course of cell fate specification causing unique responses to rather non-specific signals (Flores et. al. 2000, Tsuda et al., 2002).

\section{References}

ALAWI, A. A., JENNINGS, V., GROSSFIELD, J. and PAK, W. L. (1972). Phototransduction mutants of Drosophila melanogaster. Adv Exp Med Biol24, $1-21$.

AROIAN, R. V., KOGA, M., MENDEL, J. E., OHSHIMA, Y. and STERNBERG, P. W. (1990). The let-23 gene necessary for Caenorhabditis elegans vulval induction encodes a tyrosine kinase of the EGF receptor subfamily. Nature 348, 693-9.

BAKER, N. E. and RUBIN, G. M. (1989). Effect on eye development of dominant mutations in Drosophila homologue of the EGF receptor. Nature 340, 150-3.

BAKER, N. E. and RUBIN, G. M. (1992). Ellipse mutations in the Drosophila homologue of the EGF receptor affect pattern formation, cell division, and cell death in eye imaginal discs. Dev Bio/150, 381-96.

BAKER, W. (1963). Genetic control of pigment differentiation in somatic cells. Am. Zoo/3, 57-69.

BANERJEE, U., RENFRANZ, P. J., HINTON, D. R., RABIN, B. A. and BENZER, S. (1987a). The sevenless ${ }^{+}$protein is expressed apically in cell membranes of developing Drosophila retina; it is not restricted to cell R7. Ce//51, 151-8.

BANERJEE, U., RENFRANZ, P. J., POLLOCK, J. A. and BENZER, S. (1987b). Molecular characterization and expression of sevenless, a gene involved in neuronal pattern formation in the Drosophila eye. Cel/49, 281-91.

BASLER, K., CHRISTEN, B. and HAFEN, E. (1991). Ligand-independent activation of the sevenless receptor tyrosine kinase changes the fate of cells in the developing Drosophila eye. Cel/64, 1069-81.

BASLER, K. and HAFEN, E. (1988). Sevenless and Drosophila eye development: a tyrosine kinase controls cell fate. Trends Genet 4, 74-9.
BERNARD, F. (1937). Recherches sur la morphogenese des yeux composes d'arthropodes. Bull.Biol.Fr.Belg (suppl) 23, 3-162.

BIGGS, W. H., 3RD, ZAVITZ, K. H., DICKSON, B., VAN DER STRATEN, A., BRUNNER, D., HAFEN, E. and ZIPURSKY, S. L. (1994). The Drosophilarolled locus encodes a MAP kinase required in the sevenless signal transduction pathway. EMBO J13, 1628-35.

BIGGS, W. H., 3rd and ZIPURSKY, S. L. (1992). Primary structure, expression, and signal-dependent tyrosine phosphorylation of a Drosophila homolog of extracellular signal-regulated kinase. Proc Nat/ Acad Sci USA 89, 6295-9.

BONFINI, L., KARLOVICH, C. A., DASGUPTA, C. and BANERJEE, U. (1992). The Son of sevenless gene product: a putative activator of Ras. Science 255, 6036.

BRUNNER, D., OELLERS, N., SZABAD, J., BIGGS, W. H., 3RD, ZIPURSKY, S. L. and HAFEN, E. (1994). A gain-of-function mutation in Drosophila MAP kinase activates multiple receptor tyrosine kinase signaling pathways. Cel/76, 875-88.

DIAZ-BENJUMEA, F.J., and HAFEN, E (1994). The sevenless signaling cassette mediates Drosophila EGF receptor function during epidermal development. Development 120, 569-78.

DICKSON, B., SPRENGER, F., MORRISON, D. and HAFEN, E. (1992). Raf functions downstream of Ras 1 in the Sevenless signal transduction pathway. Nature 360, 600-3.

FLORES, G. V., DUAN, H., YAN, H., NAGARAJ, R., FU, W., ZOU, Y., NOLL, M. and BANERJEE, U. (2000). Combinatorial signaling in the specification of unique cell fates. Cel/103, 75-85.

FREEMAN, M. (1996). Reiterative use of the EGF receptor triggers differentiation of all cell types in the Drosophila eye. Cel/87, 651-60.

GAUL, U., MARDON, G. and RUBIN, G. M. (1992). A putative Ras GTPase activating protein acts as a negative regulator of signaling by the Sevenless receptor tyrosine kinase. Cel/68, 1007-19.

HAFEN, E., BASLER, K., EDSTROEM, J. E. and RUBIN, G. M. (1987). Sevenless a cell-specific homeotic gene of Drosophila, encodes a putative transmembrane receptor with a tyrosine kinase domain. Science 236, 55-63.

HAN, M., GOLDEN, A., HAN, Y. and STERNBERG, P. W. (1993). C. elegans lin45 raf gene participates in let-60 ras-stimulated vulval differentiation. Nature $363,133-40$.

HAN, M. and STERNBERG, P. W. (1990). let-60, a gene that specifies cell fates during $C$. elegans vulval induction, encodes a ras protein. Ce//63, 921-31.

HARRIS, W. A. and STARK, W. S. (1977). Hereditary retinal degeneration in Drosophilamelanogaster. A mutant defect associated with the phototransduction process. J Gen Physio/69, 261-91.

HARRIS, W. A., STARK, W. S. and WALKER, J. A. (1976). Genetic disscection of the photoreceptor system in the compound eye of Drosophila melanogaster. J.Physiol. 256, 415-439.

HART, A. C., KRAMER, H., VAN VACTOR, D. L., JR., PAIDHUNGAT, M. and ZIPURSKY, S. L. (1990). Induction of cell fate in the Drosophila retina: the bride of sevenless protein is predicted to contain a large extracellular domain and seven transmembrane segments. Genes Dev 4, 1835-47.

HILL, R. J. and STERNBERG, P. W. (1992). The gene lin-3 encodes an inductive signal for vulval development in C. elegans. Nature 358, 470-6.

HOTTA, Y. and BENZER, S. (1969). Abnormal electroretinograms in visual mutants of Drosophila. Nature 222, 354-6.

HOTTA, Y. and BENZER, S. (1970). Genetic dissection of the Drosophilanervous system by means of mosaics. Proc Nat/ Acad Sci USA 67, 1156-63.

KENYON, K. L., RANADE, S. S., CURTISS, J., MLODZIK, M. and PIGNONI, F. (2003). Coordinating proliferation and tissue specification to promote regional identity in the Drosophila head. Dev. Cel/5, 403-14

KOCH, C. A., ANDERSON, D., MORAN, M. F., ELLIS, C. and PAWSON, T. (1991). $\mathrm{SH} 2$ and $\mathrm{SH} 3$ domains: elements that control interactions of cytoplasmic signaling proteins. Science 252, 668-74.

KOENIG, J. and MERRIAM, J. R. (1975). Autosomal ERG mutants. Drosoph. info. Serv.

KUMAR, J. and MOSES, K. (1997). Transcription factors in eye development: a gorgeous mosaic? Genes Dev11, 2023-8.

KUMAR, J. and MOSES, K (2001) EGF Receptor and Notch signaling act upstream of Eyeless/Pax6 to control eye specification. Cel/104, 687-97. 
LAWRENCE, P. A. and GREEN, S. M. (1979). Cell lineage in the developing retina of Drosophila. Dev Bio/71, 142-52.

MOSES, K, editor (2002). Drosophila eye development. Springer-Verlag, Berlin

OLIVIER, J. P., RAABE, T., HENKEMEYER, M., DICKSON, B., MBAMALU, G., MARGOLIS, B., SCHLESSINGER, J., HAFEN, E. and PAWSON, T. (1993). A DrosophilaSH2-SH3 adaptor protein implicated in coupling the sevenless tyrosine kinase to an activator of Ras guanine nucleotide exchange, Sos. Cel/73, 179-91.

ONUMA, Y., TAKAHASHI, S., ASASHIMA, M., KURATA, S., GEHRING, W.J., (2002). Conservation of D-Pax6 function and upstream activation by Notch in eye development in Frogs and flies Proc Nat/ Acad Sci USA 99, 2020-25

PAK, W. L. and LEUNG, H. T. (2003). Genetic approaches to visual transduction in Drosophila melanogaster. Receptor Channels 9, 149-67.

PAPPU, K., MARDON, G. (2002) Retinal specification and determination in Drosophila. In Drosophila Eye Development Ed K, Moses. 5-20.

PAWSON, T. (2002). Regulation and targets of receptor tyrosine kinases. Eur J. Cancer Suppl 5, 3-10.

PERRY. (1968). Further studies on the development of the eye of Drosophila melanogaster II: The ommatidia. J.Morphology 124, 249-62.

READY, D. F., HANSON, T. E. and BENZER, S. (1976). Development of the Drosophila retina, a neurocrystalline lattice. Dev Bio/53, 217-40.

REINKE, R and ZIPURSKY, S.L. (1988) Cell-cell interaction in the Drosophila eye: the bride of sevenless gene is required in photoreceptor cell R8 for R7 cell development. Cel/55: 321-330.

ROGGE, R., CAGAN, R., MAJUMdAR, A., DULANEY, T. and BANERJEE, U. (1992). Neuronal development in the Drosophilaretina: the sextra gene defines an inhibitory component in the developmental pathway of R7 photoreceptor cells. Proc Nat/ Acad Sci USA 89, 5271-5.

ROGGE, R. D., KARLOVICH, C. A. and BANERJEE, U. (1991). Genetic dissection of a neurodevelopmental pathway: Son of sevenless functions downstream of the sevenless and EGF receptor tyrosine kinases. Ce//64, 39-48.
SHOUP, J. (1966). The development of pigment granules in the eye of wild type and mutant Drosophila melanogaster. J. Cell Biology 29, 223-249.

SIMON, M. A., BOWTELL, D. D., DODSON, G. S., LAVERTY, T. R. and RUBIN, G. M. (1991). Ras1 and a putative guanine nucleotide exchange factor perform crucial steps in signaling by the sevenless protein tyrosine kinase. Cel/67, 701-16.

SIMON, M. A., DODSON, G. S. and RUBIN, G. M. (1993). An SH3-SH2-SH3 protein is required for p21Ras1 activation and binds to sevenless and Sos proteins in vitro. Cel/73, 169-77.

TOMLINSON, A. and READY, D. F. (1986). sevenless, a cell specific homeotic mutation of the Drosophila eye. Science 231, 400-402.

TOMLINSON, A. and READY, D. F. (1987). Neuronal differentiation in Drosophila ommatidium. Dev Bio/120, 336-376.

TSUDA, L., NAGARAJ, R., ZIPURSKY, S. L. and BANERJEE, U. (2002). An EGFR/ Ebi/Sno pathway promotes delta expression by inactivating $\mathrm{Su}(\mathrm{H}) / \mathrm{SMRTER}$ repression during inductive notch signaling. Cel/110, 625-37.

VAN VACTOR, D. L., JR., CAGAN, R. L., KRAMER, H. and ZIPURSKY, S. L. (1991). Induction in the developing compound eye of Drosophila: multiple mechanisms restrict R7 induction to a single retinal precursor cell. Cel/67, 1145-55.

WADDINGTON, C. H. and PERRY, M. M. (1961). The ultra structure of the developing eye of Drosophila. Philos Trans R Soc Lond B Bio/Sci153, 155-178.

WOLFF, T. and READY, D. F. (1991). The begining of pattern formation in the Drosophila compound eye: The morphogenetic furrow and the second wave. Development 113, 841-850.

WOLFF, T. and READY, D. F. (1993) Patten formation in the Drosophila retina. In: Bates M, Arias, AM (ed) Cold Spring Harbor laboratatory Press, NY 12771326

XU, C., KAUFFMANN, R. C., ZHANG, J., KLADNY, S. and CARTHEW, R. W. (2000). Overlapping activators and repressors delimit transcriptional response to receptor tyrosine kinase signals in Drosophila eye. Cel/103, 87-97. 